\title{
Seroprevalence of Dengue Virus in Hidalgo County from 2005-2017
}

\author{
Laiba Asif ${ }^{1}$, Juan Garcia ${ }^{1}$, Steven Hinojosa ${ }^{3}$, Christopher J Vitek ${ }^{1}$, John M Thomas III ${ }^{1,2 *}$ \\ ${ }^{1}$ The University of Texas Rio Grande Valley, Edinburg, USA \\ ${ }^{2}$ The University of Texas Rio Grande Valley School of Medicine, Edinburg, USA
}

${ }^{3}$ Hidalgo County Health Department, Edinburg, USA

*Corresponding author: John M Thomas, The University of Texas Rio Grande Valley, Edinburg, Texas, The University of Texas Rio Grande Valley School of Medicine, Edinburg, Texas, USA

\author{
ARTICLE INFO \\ Received: webruary 13, 2020 \\ Published: 㗀 February 20, 2020 \\ Citation: Laiba A, Juan G, Steven H, \\ Christopher J Vitek, John M Thomas III. \\ Seroprevalence of Dengue Virus in Hidalgo \\ County from 2005-2017. Biomed J Sci \& \\ Tech Res 25(5)-2020. BJSTR. MS.ID.004264.
}

ABSTRACT

Keywords: Dengue; Texas; Surveillance; Mosquito; Testing

\section{Short Communication}

We report the testing results for Dengue in Hidalgo County, Texas from 2005-2017. Serology and PCR testing of submitted samples confirmed that, out of 121 submitted samples, 18 ( 15\%) were positive for Dengue virus. The highest number of positive samples were from Edinburg and Weslaco, Texas. Dengue virus (DENV) is single-stranded, positive-sense arbovirus that belongs to the family Flaviviridae. This family includes other medically significant viruses such as West Nile virus and Zika virus. There are four antigenically different serotypes of DENV: DENV-1, DENV-2, DENV-3, and DENV-4. All four serotypes can be found worldwide, posing a threat to one-third of the global population and causing an estimated 390 million new infections every year [1]. DENV has historically been transmitted throughout northern Mexico, but few studies have been conducted near the northern Mexico-south Texas border. There is an extreme disparity between reported cases of dengue virus in the northern border states of Mexico, and in the border regions of South Texas [2].

From 1980-1999, the border states of Mexico reported 64,514 cases of DENV, whereas Texas only reported 64 cases [3]. Recent studies suggest that DENV has been substantially underreported on both sides of the border [4]. Evidence suggests that DENV poses a risk to populations in the Lower Rio Grande Valley (LRGV) of South Texas (composed of Cameron, Hidalgo, and Starr counties), which borders directly with northern Mexico. Both northern Mexico and the LRGV share similar subtropical climates and an abundance of Aedes aegypti and Aedes albopictus vectors for dengue virus [2,5]. DENV risk is also associated with family income, suggesting that economically depressed unincorporated regions of South Texas (colonies) where individuals often lack access to air conditioning and screened windows that typically prevent or reduce humanmosquito contact may be at higher risk [1,6]. In 2004, a crosssectional survey was conducted between the cities of Brownsville and Matamoros to determine the population seroprevalence of DENV [4].

Evidence of recent DENV infection was found in $2 \%$ of Brownsville residents, and historical evidence of past DENV infection was found in $40 \%$ of Brownsville residents. In 2005, a household-based serological study was conducted, estimating the incidence of re- 
cent infection at $4 \%$ and the prevalence of past infection at $39 \%$ of Brownsville residents [7]. These seroprevalence values suggested that the number of DENV infections in border cities was large and likely to increase with each DENV epidemic in Northern Mexico. Unfortunately, there is no current data on the seroprevalence of DENV in the LRGV, and information has been limited to the Cameron county area. The purpose of this study was to examine the seroprevalence of DENV in Hidalgo County, Texas between the years 2005-2017. Data was acquired from antibody testing results from human clinical samples submitted to the Hidalgo County Health Department (HCHD) and confirmed using RT-PCR by Texas DSHS. A total of 121 samples were screened from 2005-2017.

Approximately $15 \%$ of the samples submitted tested positive for DENV spread across the 12-year timeframe, with smaller municipalities such as La Joya, Elsa, Pharr, and Palmview having a higher percentage of positive samples. The larger cities in Hidalgo County: McAllen, Edinburg, and Mission had more testing done than the smaller municipalities such as Alton, La Joya, La Blanca, and La Villa however, 12/18 municipalities had at least one DENVpositive case during the testing period. In smaller cities such as Alton, La Blanca, La Joya, La Villa, and Palmview, DENV testing from 2005-2017 was often confined to a single calendar year, whereas all other cities showed testing activities spread across the 20052017 time period. It is possible that the presence of a large number of lower-income dwellings such as colonias in and around Hidalgo County may facilitate vector breeding and human exposure due to the presence of viable oviposition habitat, and the likelihood for household-household transmission of DENV [5] (Figure 1).

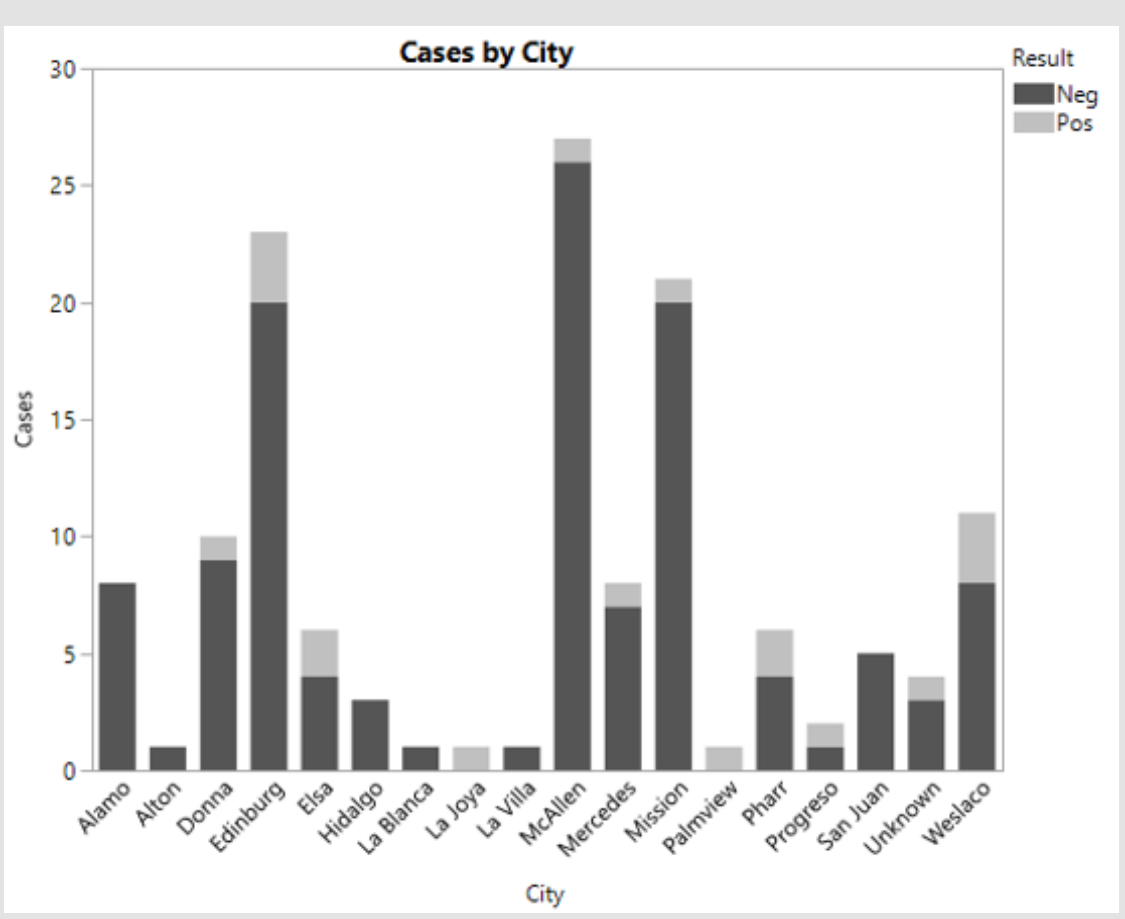

Figure 1: Total cases (by city) of suspected DENV submitted to Hidalgo County Health Department from years $2005-2017$. Samples submitted for testing showed that larger cities with some degree of systemic surveillance had more submissions than smaller cities however, 12/18 municipalities had at least (1) DENV+ case during the testing period.

Surveillance of mosquito-borne diseases in the United States has been limited in both scope and application, as these activities are often funded by local municipalities. However, other countries where robust surveillance programs exist use a combined approach of pathogen-specific physician education coupled with year-round mosquito trapping and rapid response once cases are identified as positive [8]. In south Texas, efforts to increase public awareness of the incidence of febrile disease and the potential connection between fever and arboviral infection has resulted in local governmental entities such as HCHD directing cases of febrile disease to local health professionals for arbovirus testing, particularly DENV. The sample population tested for DENV exhibits some bias as it was limited to individuals who self-reported to the HCHD, and it is likely that many suspected DENV cases were unaccounted for due to a lack of reporting.

\section{Acknowledgement}

We thank our partners at the Hidalgo County Health Department for providing the testing data and methodology.

\section{Conflict of Interest}

The authors declare no competing conflicts of interest.

\section{References}

1. Brunkard JM, Lopez JLR, Ramirez J, Cifuentes E, Rothenberg SJ, et al. (2007) Seroprevalence and risk factors for dengue fever on the TexasMexico border, 2004. Emerging Infectious Diseases 13: 1477-1483. 
2. Ferreira-de-Lima VH, Lima-Camara TN (2018) Natural vertical transmission of dengue virus in Aedes aegypti and Aedes albopictus: a systematic review. Parasites and Vectors 11(1): 77.

3. Joshi V, Mourya DT, Sharma RC (2002) Persistence of dengue-3 virus through transovarial transmission passage in successive generations of Aedes aegypti mosquitoes. American Journal of Tropical Medicine and Hygiene 67(2): 158-161.

4. Lequime S, Lambrechts L (2014) Vertical transmission of arboviruses in mosquitoes: a historical perspective. Infection, Genetics, and Evolution 28: 681-690.

5. Vitek CJ, Gutierrez JA, Dirrigl FJ (2014) Dengue vectors, human activity, and dengue virus transmission potential in the Lower Rio Grande Valley, Texas, United States. Journal of Medical Entomology 51: 1019-1028.

\section{ISSN: 2574-1241}

DOI: 10.26717/BJSTR.2020.25.004264

John M Thomas. Biomed J Sci \& Tech Res

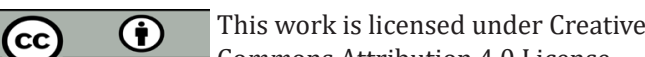

Submission Link: https://biomedres.us/submit-manuscript.php
6. Ramos MM, Mohammed H, Zielinski-Gutierrez E, Hayden MH, Lopez JLR, et al. (2008) Epidemic dengue and dengue hemorrhagic fever at the Texas-Mexico border: results of a household-based seroepidemiologic survey, December 2005. American Journal of Tropical Medicine and Hygiene 78(3): 364-369.

7. Reiter P, Lathrop S, Bunning M, Biggerstaff B, Singer D, et al. (2003) Texas lifestyle limits transmission of dengue virus. Emerging Infectious Diseases 9(1): 86-89.

8. Andersson N, Nava Aguilera E, Arosteguí J, Morales-Perez A, SuazoLaguna H, et al. (2015) Evidence based community mobilization for dengue prevention in Nicaragua and Mexico (Camino Verde, the Green Way): cluster randomized controlled trial. BMJ 351: h3267.

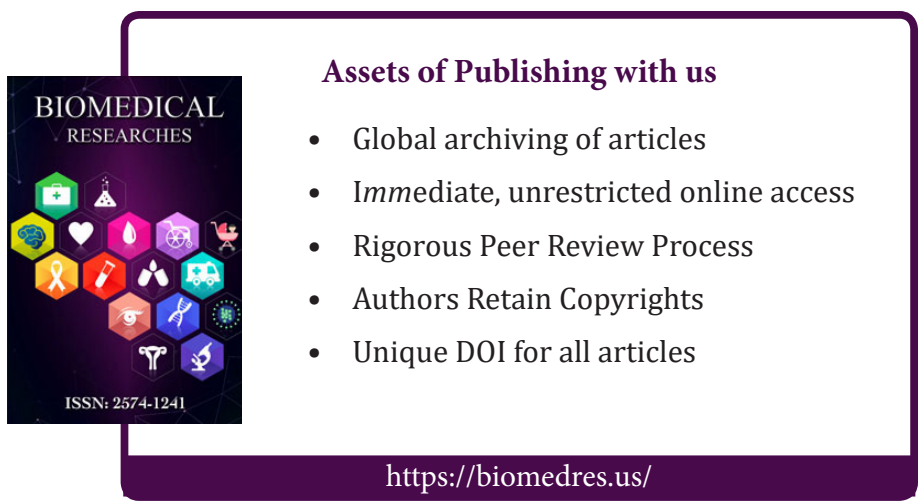

